Supporting Information for

\title{
Realization of Superhydrophobic Surface for the Detection of Residual Detergent Concentration
}

\author{
Eunbin Cho, ${ }^{\dagger}$ Gitae Namgung, ${ }^{\dagger}$ Chulkyoon Mok,,${ }^{\ddagger}$ and Jin-Seo Noh*,† \\ $\dagger^{\dagger}$ Department of Nano-Physics, Gachon University, 1342 Seongnamdaero, Sujeong-gu, \\ Seongnam-si, Gyeonggi-do 13120, Korea \\ ¥Department of Food Science and Biotechnology, Gachon University, 1342 Seongnamdaero, \\ Sujeong-gu, Seongnam-si, Gyeonggi-do 13120, Korea \\ *Author to whom correspondence should be addressed: Jin-Seo Noh: \\ e-mail: jinseonoh@gachon.ac.kr, \\ phone: +82317505611
}

Number of pages: 9

Number of figures: 8

Number of tables: 0 

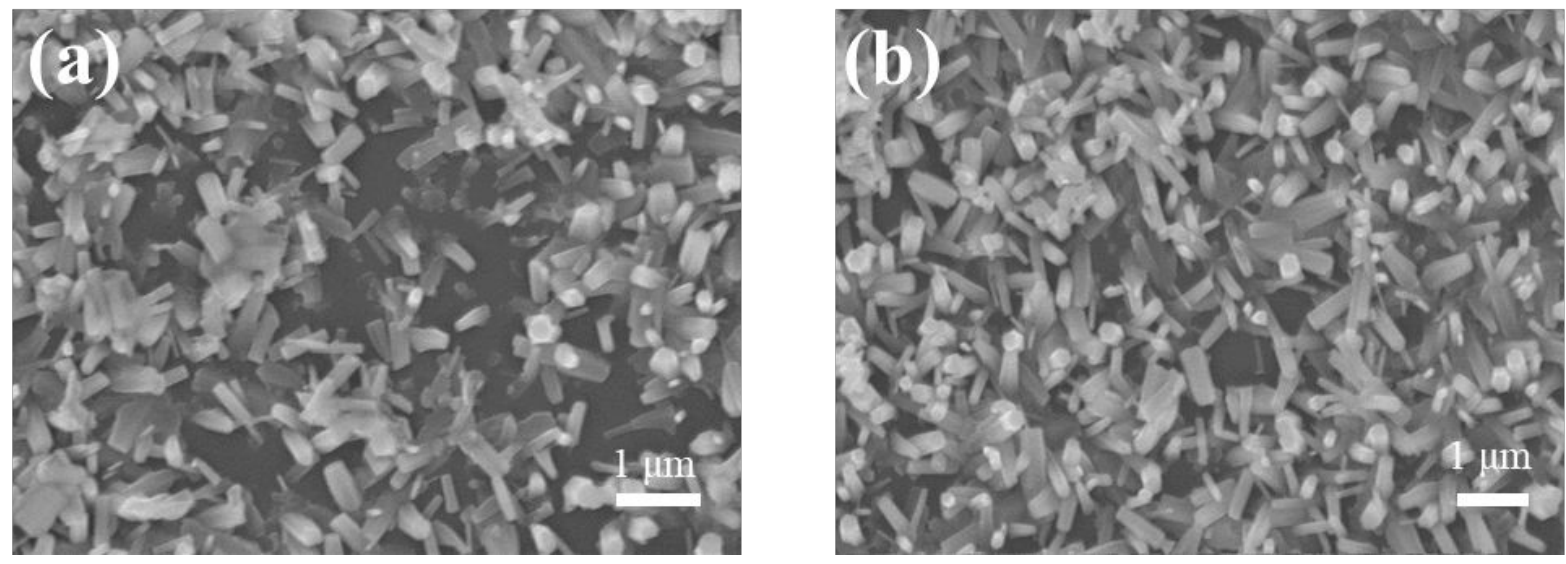

Figure S1. SEM images of $\mathrm{ZnO}$ Nanorods grown on the glass substrate without seed layer formation. The two images were obtained from different locations on the same sample. 


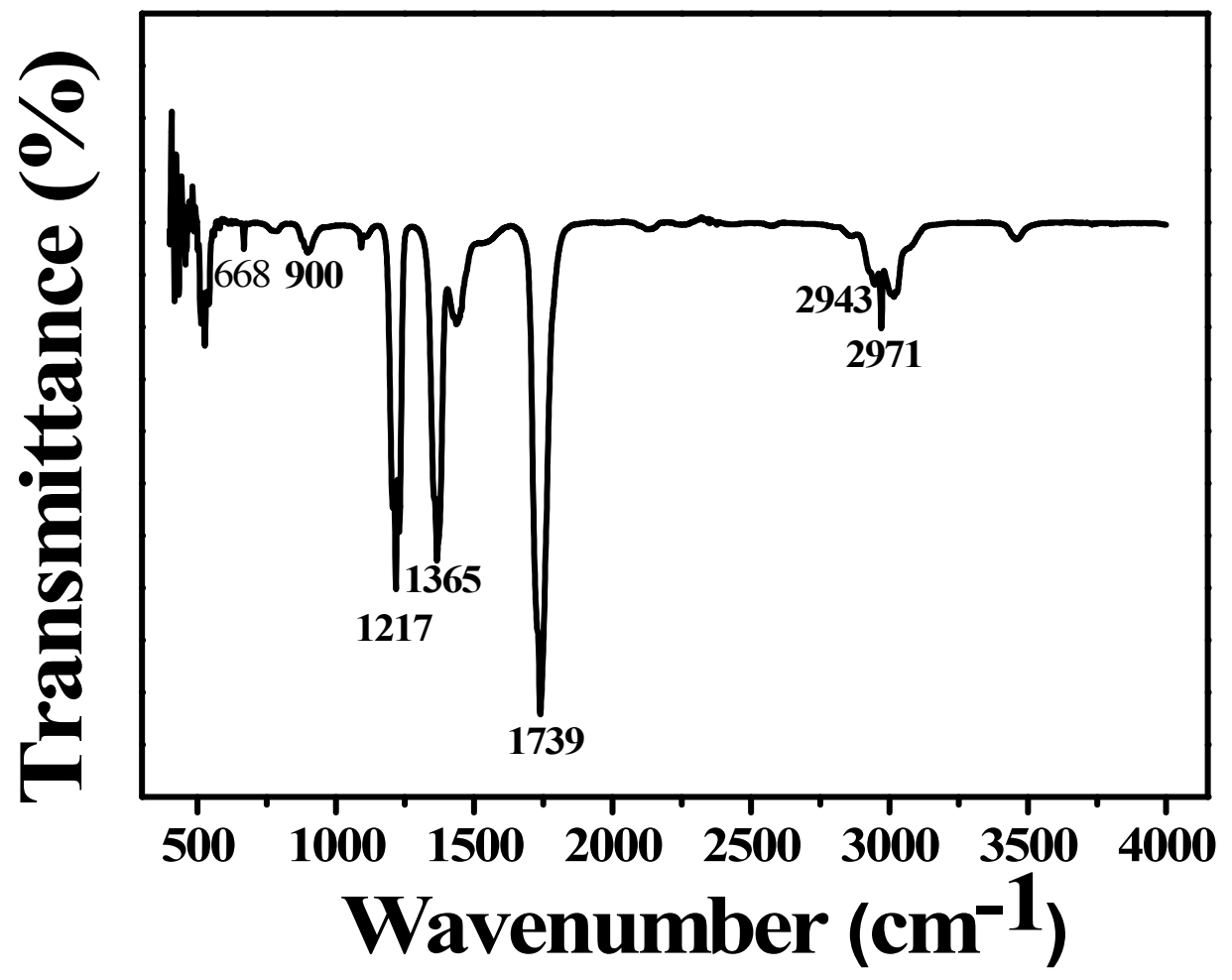

Figure S2. FT-IR spectrum of pure stearic acid solution. 
(a) Bare glass

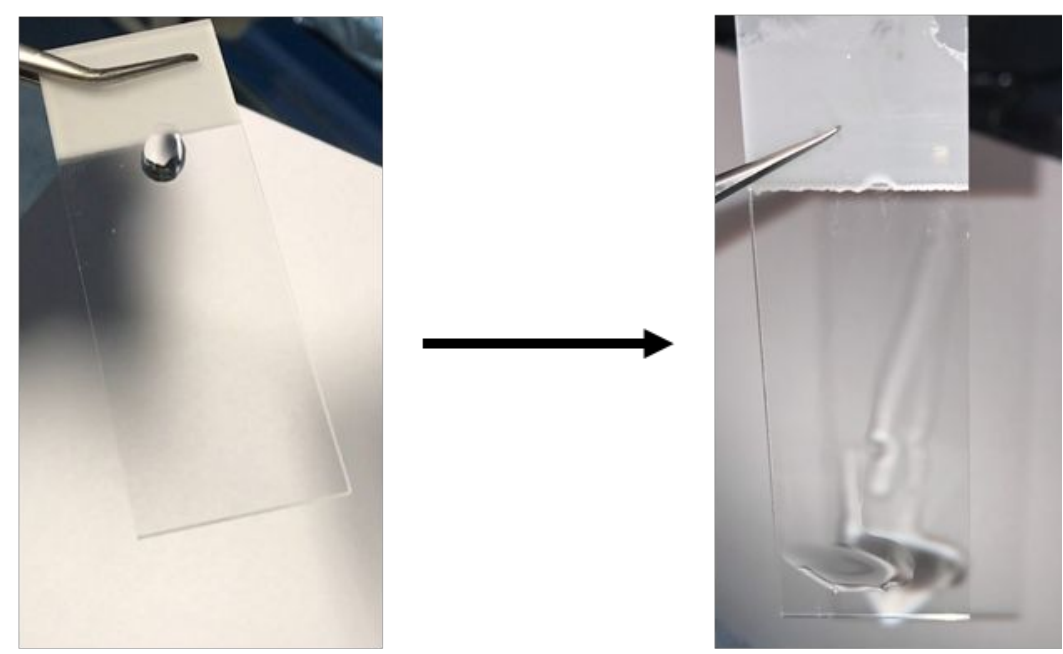

\section{(b) Stearic acid-treated $\mathrm{ZnO}$ nanorods}
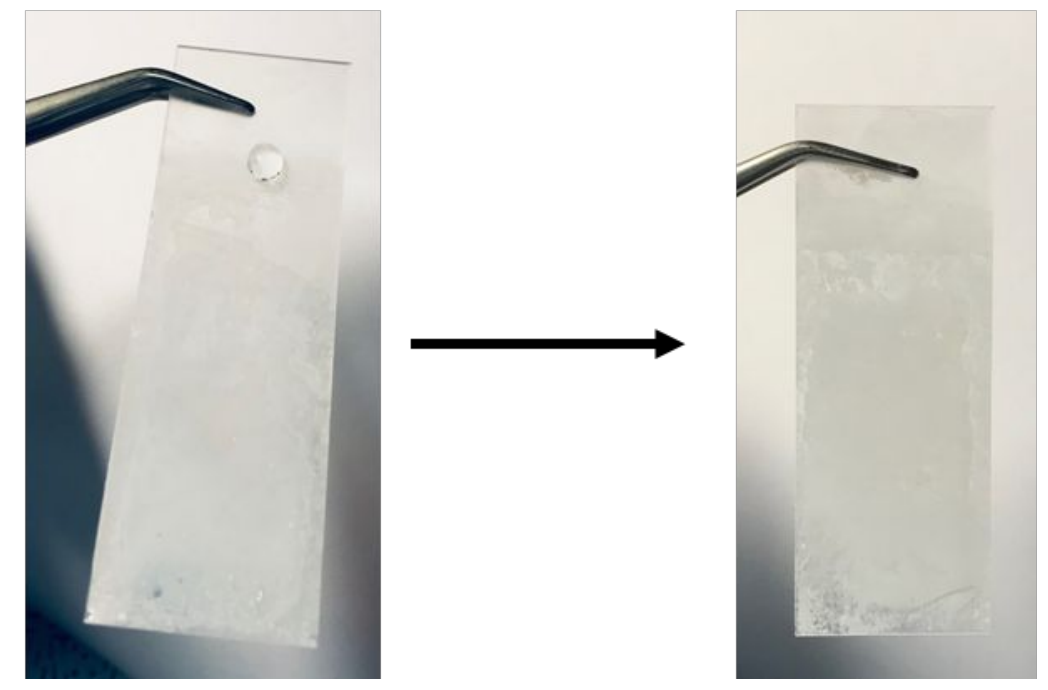

Figure S3. Images of water drops (a) on the bare glass and (b) on the surface-modified glass using stearic acid-treated $\mathrm{ZnO}$ nanorods. At a tiled angle, water drop on the bare glass spreads over the large area, whereas it rapidly rolls off the surface-modified glass without spreading. 

(a) 1 ppm

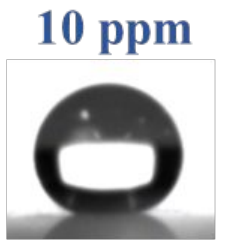
100 ppm
1000 ppm
$\theta=152.8^{\circ}$
$\theta=151.7^{\circ}$
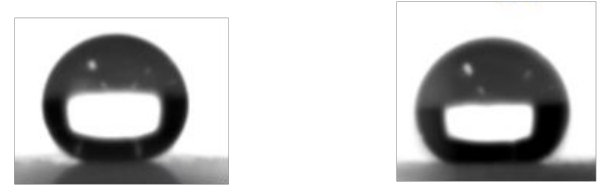
$\theta=150.0^{\circ}$
$\theta=144.3^{\circ}$
(b)
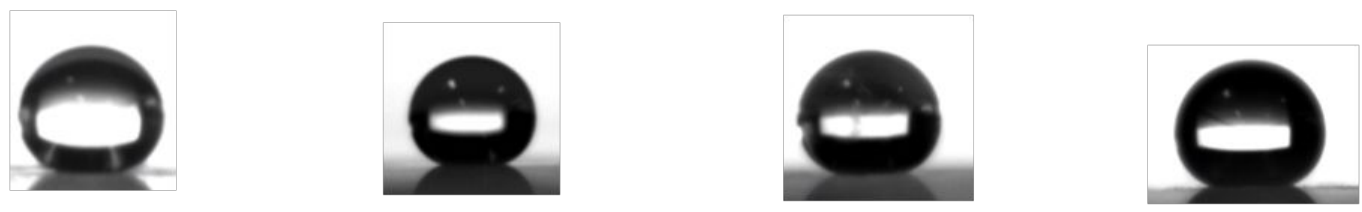
$\theta=150.0^{\circ}$
$\theta=148.0^{\circ}$
$\theta=147.7^{\circ}$
$\theta=142.9^{\circ}$
(c)
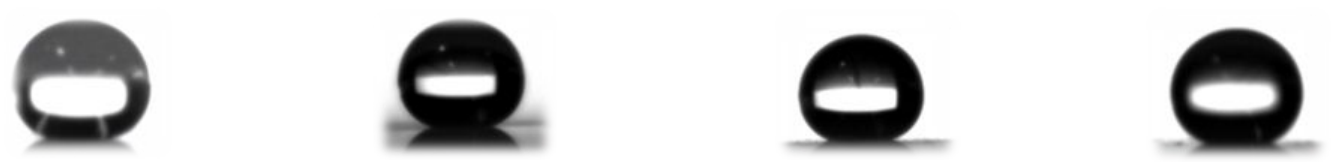

$$
\theta=150.0^{\circ}
$$
$\theta=143.3^{\circ}$
$\theta=141.9^{\circ}$
$\theta=137.5^{\circ}$

Figure S4. Images and average contact angles of detergent solutions on the surface-modified glass: (a) rinse aid dishwasher, (b) sodium detergent, and (c) potassium detergent. The contact angles were measured as a function of detergent concentration. 


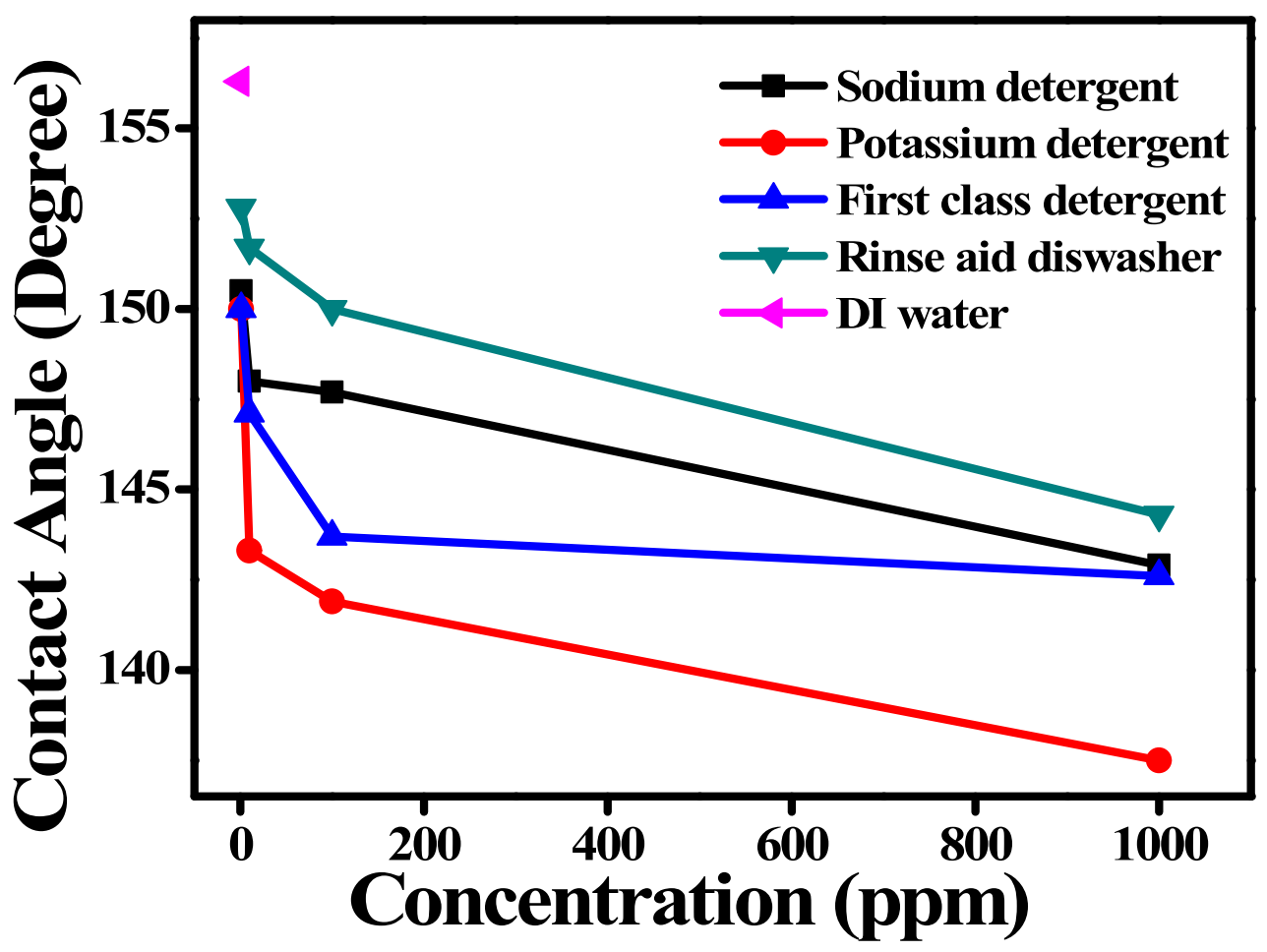

Figure S5. Contact angle versus detergent concentration curves for different types of detergents. 
(a)
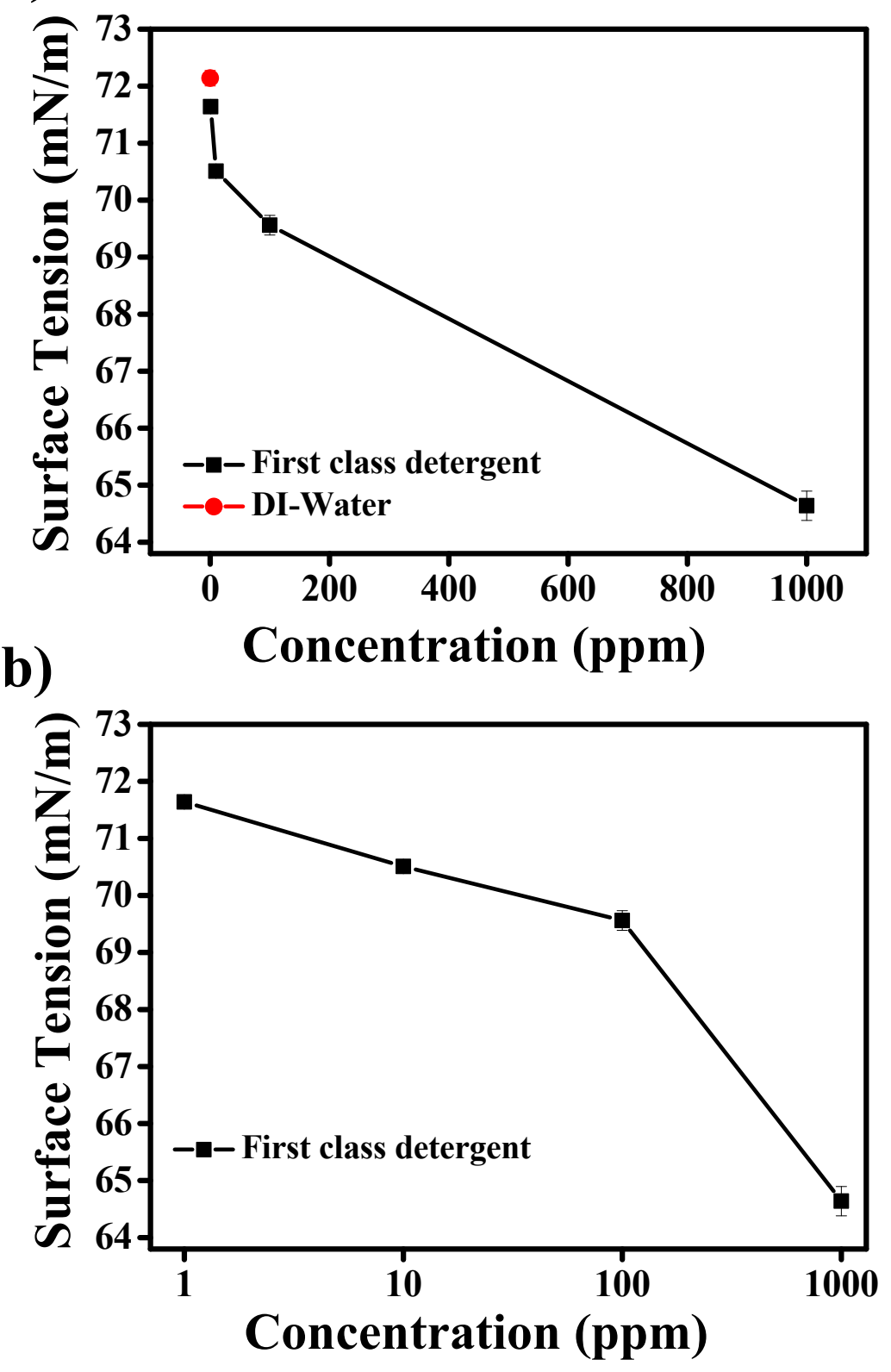

Figure S6. (a) Surface tension versus concentration curve for DI water and first class detergent solutions. (b) Semi-log plot between the surface tensions and detergent concentrations for the first class detergent solutions. 

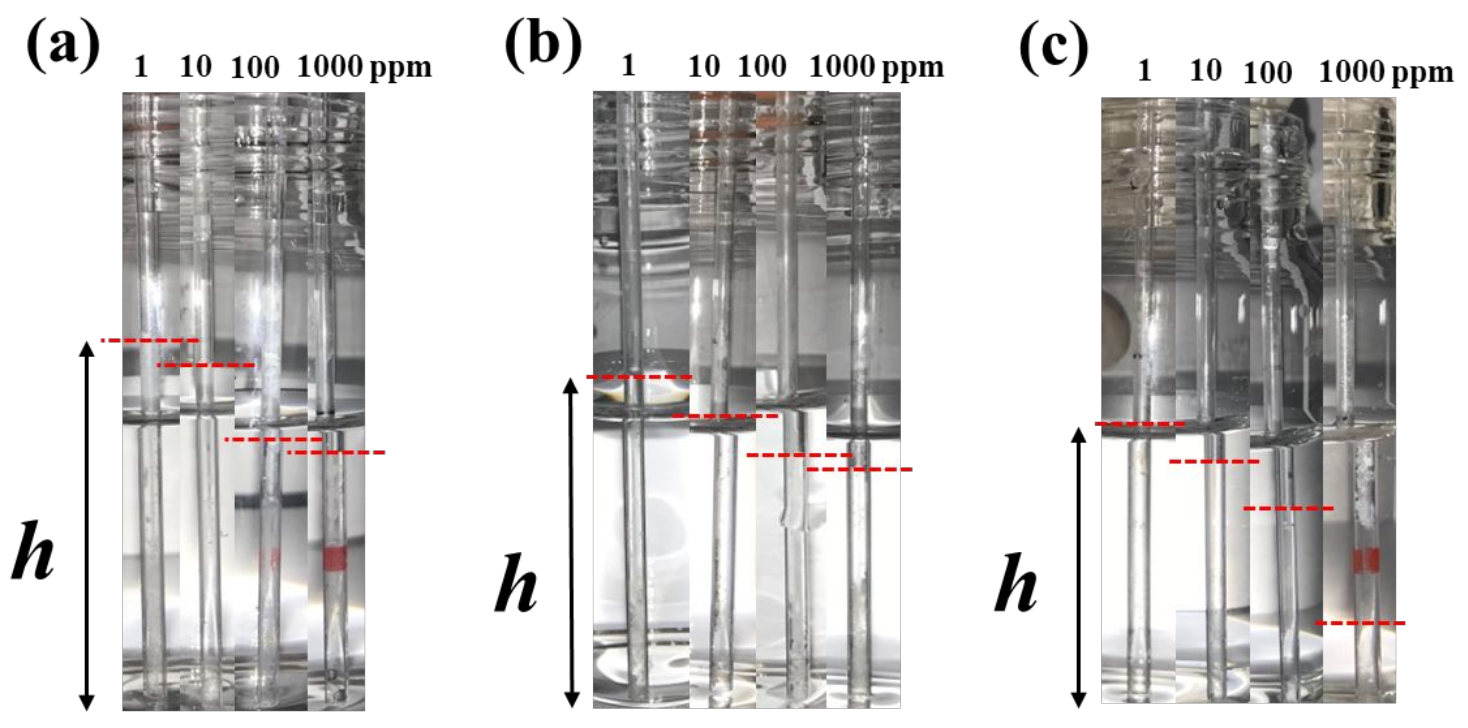

Figure S7. Detergent concentration-dependent capillary heights for different detergent solutions: (a) rinse aid dishwasher, (b) sodium detergent, and (c) potassium detergent. 


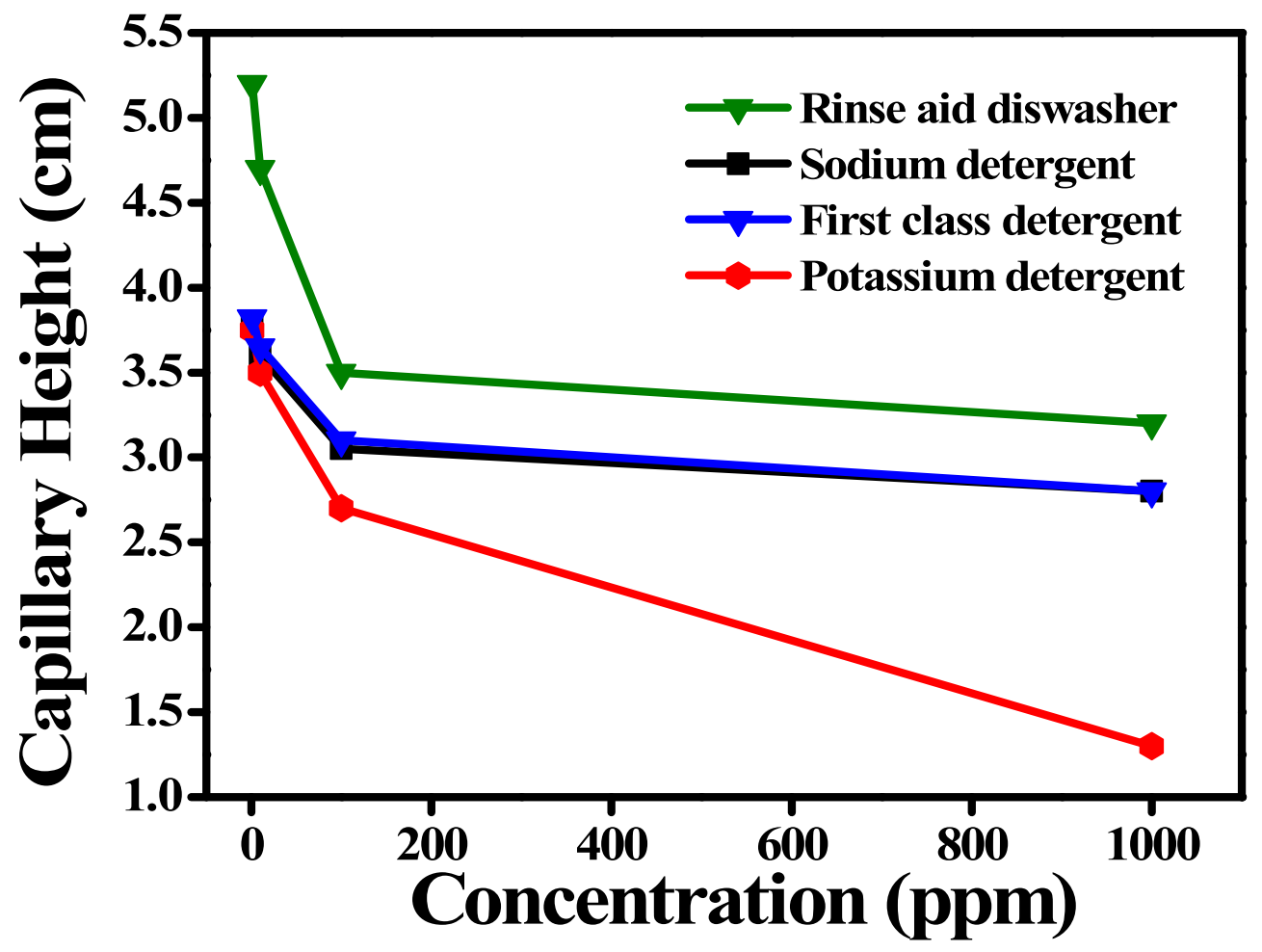

Figure S8. Capillary height versus detergent concentration curves for different types of detergents. 